\title{
Über die Trennung des Zinks von anderen Metallen, insbesonders von Nickel.
}

\author{
Von Alois Ludwig.
}

Ich habe in vorliegender Arbeit versucht, das Anwendungsgebiet von Neutralsalzen der Weinsäure und Rhodanwasserstoffsüure (speziell deren Ammonsalze) zu erweitern und die Möglichkeit der Verwendung derselben zur quantitativen Trennung. des Zinks von Nickel zu prüfen. Ich konnte feststellen, daß die in dieser Richtung angestellten Versuche zur Ausarbeitung brauchbarer Methoden führten. und zwar:

1. Trennung des Zn von Ni mit Sehwefelwasserstoff aus schwachmineralsaurer, ammontartrathaltiger Lösung der beiden Metalle und

2. Trennung des $\mathrm{Zn}$ von $\mathrm{Ni}$ mit $\left(\mathrm{NH}_{4}\right)_{2} \mathrm{HPO}_{4}$, wobei das $\mathrm{Zn}$ als $\mathrm{ZnNH}_{4} \mathrm{PO}_{4}$ gefällt wird, $\mathrm{Ni}$ jedoch durch neutrale Tartrate oder Ammonrhodanid durch Komplexsalzbildung in Lösung bleibt.

Zu 1. Während Sмiтh und Brunner ${ }^{1}$ ) bei der Trennung von $\mathrm{Ni}$ mit $\mathrm{H}_{2} \mathrm{~S}$ das Ammon- bzw. Alkalisalz der schwachen Essigsäure, hingegen Zimmermann ${ }^{2}$ und G. H. Kramers ${ }^{3}$ die Ammonsalze der starken Säuren (HCNS bzw. $\mathrm{H}_{2} \mathrm{SO}_{4}$ oder $\mathrm{HCl}$ ) verwenden, benütze ich das neutrale Ammonsalz der Weinsäure, welche ungefähr in der Mitte zwischen schwachen und starken Säuren steht, eher aber als schwache Säure anzusprechen ist.

$\mathrm{Zu}$ 2. Für die Bestimmung des Zinks als $\mathrm{ZnNH}_{4} \mathrm{PO}_{4}$ und die hierauf basierende Trennung von Nickel untersuchte ich in einer Reihe von Vorversuchen den Einfluß der Neutralsalze auf diese Fällung und gelangte zu folgendem Ergebnis:

Das neutrale Ammontartrat bzw. neutrale Alkalitartrate und Ammonrhodanid, welche die Fällung des Zinks als $\mathrm{ZnNH}_{4} \mathrm{PO}_{4}$ nicht oder nur wenig störend beeinflussen, bilden bei genügendem $\mathrm{Zu}$ satz mit Nickelsalzen derart beständige komplexe Verbindungen, so daß bei Zugabe von $\left(\mathrm{NH}_{4}\right)_{2} \mathrm{HPO}_{4}$ zu solchen Ni-Lösungen auch

1) Chem. Zentrabblatt 1895, 26.

2) Ann. d. Chem. u. Pharm. 199 (1879), 3; 204 (1880), 246.

3) Traedwelu, Lehrbuch d. analyt. Chemie II. 
in der Hitze keine oder nur geringe Umsetzung unter Bildung von $\mathrm{NiNH}_{4} \mathrm{PO}_{4}$ eintritt.

Ich verwende nun diese von versehiedenen Forschern ${ }^{1}$ ) eingehend bearbeitete Fällung des Zinks als $\mathrm{ZnNH}_{4} \mathrm{PO}_{4}$ für die Trennung der beiden Metalle und erhielt durchwegs zufriedenstellende Analysenergebnisse.

Im folgenden werde ich die einzelnen Methoden noch ausführen und auf die hierbei auftretenden Fehlerquellen, Vor- und Nachteile gegenüber anderen Methoden näher eingehen.

\section{Die Trennung des Zinks von Nickel mit Schwefelwasserstoff ans ammontartrathaltiger, mineralsaurer Lösung.}

Die Fällung des Zinks als Sulfid mit $\mathrm{H}_{2} \mathrm{~S}$ beruht auf folgender Umsetzungsgleichung ${ }^{2}$ ):

$\mathrm{ZnCl}_{2}+\mathrm{H}_{2} \mathrm{~S} \rightleftharpoons \mathrm{ZnS}+2 \mathrm{HCl}$. Diese Reaktion ist umkehrbar. Aus konzentrierter Lösung wird das unvollständig oder bei Anwesenheit von genügend freier Mineralsäure gar nicht gefällt. Die Löslichkeit eines Sulfides in einer Säure ist um so größer, je größer dessen Löslichkeitsprodukt ist und je größer die Konzentration der H-Ionen ist. Leitet man nun in eine neutrale Zinksalzlösung $\mathrm{H}_{2} \mathrm{~S}$ ein, so scheidet sich wohl $\mathrm{ZnS}$ aus, aber mit der Zunahme des $\mathrm{ZnS}$ steigt auch die Konzentration der Wasserstoffionen.

Die Ausfällung ist demzufolge eine unvollständige. Um die Fällung vollständig zu machen, muß man die Anbäufung der H-Ionen verhindern, indem man die freigewordene Mineralsäure durch eine schwache Säure ersetzt, die nur wenig elektrolytisch dissoziert ist und daher eine Lösung mit wenig H-Ionen liefert. ${ }^{3}$ )

TREADWELL ${ }^{4}$ ) beschreibt die Fällung des Zinksulfids aus saurer Lösung wie folgt: Die mit $\mathrm{NH}_{3}$ fast neutralisierte Lösung versetzt man mit $\mathrm{NH}_{4} \mathrm{Cl}$ oder $\left(\mathrm{NH}_{4}\right)_{2} \mathrm{SO}_{4}$, fügt etwas $\mathrm{NH}_{4}$ oder Na-Acetat hinzu und leitet in der Wärme $\mathrm{H}_{2} \mathrm{~S}$ bis zur Sättigung ein. Nach völligem Absitzen des Niederschlages gießt man die überstehende klare Lösung durch ein Filter, spült den Niederschlag mit 2-4\% $\%$ iger, mit $\mathrm{H}_{2} \mathrm{~S}$ gesättigter Essigsäure auf das Filter und wäscht mit dieser Flüssigkeit vollständig aus. Nach dem Auswaschen wird der Nieder-

1) H. Tamm, Chem. News 24, 148; G. Lösekann u. Th. Meyer, Chem. Ztg. 1886, S. 729; M. Austin, Amer. Journ. Sci 1899 и. Z. anorg. u. allg. Chem. 22 (1900), 212; H. D. Dakin, Z. analyt. Chem. 39 (1900), 273; P. ARtmann, $Z$. analyt. Chem. 1915, 89; G. LUFF, Chem. Ztg. 77 (1921), 613; BALLEREw, Z. analyt Chem. 60 (1921), 442.

2) Traedwet., Lehrb. d. analyt. Chem. II, 1.

3) Vgl. Bruner u. Zawadzki, Chem. Zentralblatt 1910, 5.

4) Traedweli, Lehrb. d. analyt. Chem. II, 1. 
schlag getrocknet, soviel hiervon als möglich. in einen gewogenen Roseschen Tiegel gebracht, das Filter in der Platinspirale verascht und die Asche der Hauptmasse zugefügt. Nun mischt man den Niederschlag mittels eines Platindrahtes mit $1 / 3$ seiner Menge reinen Schwefels, bedeckt mit einer dünnen Schicht Schwefel, erhitzt im H-Strom und wägt nach Erkalten im H-Strom das Zinksulfid.

Ich habe bei der Fällung des Zinks als Sulfid in mineralsaurer Lösung Ammontartrat verwendet und folgende Versuchsordnung eingehalten:

Die schwachsaure Lösung von $\mathrm{ZnCl}_{2}$ oder $\mathrm{ZnSO}_{4}$ wird mit 3-5. $\mathrm{g}$ Ammontartrat $\left[\left(\mathrm{NH}_{4}\right)_{2} \mathrm{C}_{4} \mathrm{H}_{4} \mathrm{O}_{6}\right]$ versetzt, mit Wasser auf $150-200 \mathrm{ccm}$ Reaktionsvolumen (entsprechend $0,1 \mathrm{~g} \mathrm{Zn}$ der Lösung) aufgefüllt, bis etwa $70^{\circ} \mathrm{C}$ erwärmt und in der Wärme bis zur Sättigung $\mathrm{H}_{2} \mathrm{~S}$ eingeleitet. Hierauf wird $1 / 2-1$ Std. am Wasserbad von $60-70^{\circ} \mathrm{C}$ der Niederschlag absitzen lassen. Nach vollständiger Klärung der Lösung wird der Niederschlag abfiltriert und mit heißem, mit $\mathrm{H}_{2} \mathrm{~S}$ gesättigtem Wasser, das im Liter etwa $2 \mathrm{~g}$ Ammontartrat enthält, gewaschen. Die ersten $20-30 \mathrm{ccm}$ werden gesondert aufgefangen und nochmals filtriert. Der Niederschlag ist grobflockig, setzt sich gut $a b$ und läßt sich rasch filtrieren. (In beiläufig $20 \mathrm{Min}$. ist die Filtration beendet.)

Die Filtrate wurden geprüft und waren zinkfrei. ${ }^{1}$ ) Ich babe diese beiden Zinksulfidfällungen durchgeführt und gefunden, daß das $\mathrm{ZnS}$ aus Ammontartrathaltiger Lösung grobflockiger ist und sich rascher absetzt als aus Acetatlösung und die Filtrationsdauer eine geringere war.

Verwendet wurde Zinkchlorid und $\mathrm{ZnSO}_{4}$ purriss. Merk, welches in $50 \mathrm{cem}$ der Lösung etwa $0,1 \mathrm{~g} \mathrm{Zn}$ enthielt und Ammontartrat neutr. Merk.

Beleganalysen: Der Zinkgehalt der benutzten Zinklösungen wurde in dieser wie auch in den weiter unten angeführten Bestimmungen nach 3 Methoden ermittelt und zwar:

1. als Metall durch Elektrolyse nach Methode Spitzer ${ }^{2}$ ) in $\mathrm{NaOH}-\mathrm{Lösung}$,

2. als $\mathrm{ZnS}$ aus saurer Lösung mit $\mathrm{H}_{2} \mathrm{~S}$ nach der oben angeführten Methode und Bestimmung nach Rose (siehe Treadwelu),

3. als $\mathrm{ZnNH}_{4} \mathrm{PO}_{4}$ aus neutraler bzw. schwach saurer Lösung nach Zusatz von $\mathrm{NH}_{4} \mathrm{Cl}$ mit $\left(\mathrm{NH}_{4}\right)_{2} \mathrm{HPO}_{4}$ und trocknen bei 105 bis $110^{\circ} \mathrm{C}$.

1) Bei Versuch 2 und 5 enthält das Filtrat Spuren von Zinksulfid.

$\left.{ }^{2}\right)$ F. Spitzer, Z. Elektrochemie 11 (1905), 40l; vgl. Traedwhld, II. 
Der Mittelwert der drei Bestimmungen ist immer als berechneter Vergleichswert angenommen:

\begin{tabular}{|c|c|c|c|c|c|c|c|}
\hline $\begin{array}{l}7 n \\
\text { ber. }\end{array}$ & $\begin{array}{l}\text { Zusatz } \\
\text { von } \\
\text { Ammon- } \\
\text { azetat }\end{array}$ & $\begin{array}{l}\mathrm{Zn} \\
\text { gef. }\end{array}$ & $\begin{array}{l}\text { Zusatz } \\
\text { von } \\
\text { Ammon- } \\
\text { turtrat }\end{array}$ & $\begin{array}{l}\text { Zn } \\
\text { gef. }\end{array}$ & $\begin{array}{l}\text { Fehler bei } \\
\text { Ammon- } \\
\text { tartrat } \\
\text { in } g \mathrm{Z}_{\mathrm{n}}\end{array}$ & $\begin{array}{c}\text { Zn } \\
\text { im } \\
\text { Filtrat }\end{array}$ & $\begin{array}{c}\text { Reakt.- } \\
\text { Vol. }\end{array}$ \\
\hline $\begin{array}{c}0,1020 \mathrm{~g} \\
" " \\
0,0512 \mathrm{~g} \\
" \prime \\
0,0100 \mathrm{~g} \\
",\end{array}$ & $\begin{array}{c}2 \mathrm{~g} \\
", \\
" \\
" \\
1 " \\
\text { 1" }\end{array}$ & $\mid \begin{array}{ll}0,1022 & \mathrm{~g} \\
0,1024 & \mathrm{~g} \\
0,1021 \mathrm{~g} \\
0,0515 \mathrm{~g} \\
0,0510 \mathrm{~g} \\
0,0514 \mathrm{~g} \\
0,0099 \mathrm{~g} \\
0,0101 \mathrm{~g}\end{array}$ & $\begin{array}{ll}2 & \mathrm{~g} \\
5 & \mathrm{~g} \\
3 & \mathrm{~g} \\
2 & \mathrm{~g} \\
2 & \mathrm{~g} \\
2 & \mathrm{~g} \\
1 & \mathrm{~g} \\
1 & \mathrm{~g}\end{array}$ & $\begin{array}{l}0,1020 \mathrm{~g} \\
0,1018 \mathrm{~g} \\
0,1023 \mathrm{~g} \\
0,0514 \mathrm{~g} \\
0,051,9 \mathrm{~g} \\
0,0511 \mathrm{~g} \\
0,0102 \mathrm{~g} \\
0,0100 \mathrm{~g}\end{array}$ & $\begin{array}{c}-\overline{0002} \mathrm{~g} \\
+0,0003 \mathrm{~g} \\
+0,0002 \mathrm{~g} \\
-0,0003 \mathrm{~g} \\
-0,0001 \mathrm{~g} \\
+0,0002 \mathrm{~g} \\
\sim\end{array}$ & $\begin{array}{l}\stackrel{\sim}{\text { Spur }} \underset{\sim}{\sim} \\
\underset{\text { Spur }}{\sim} \\
\sim \\
\sim\end{array}$ & $\begin{array}{c}150-200 \mathrm{ccm} \\
" \\
100-150 \mathrm{ccm} \\
" \\
100 " \\
",\end{array}$ \\
\hline
\end{tabular}

Die Trennung des Zinks von Nickel mit Schwefelwasserstoff beruht auf der geringen Löslichkeit des Zinksulfids und der leichten Löslichkeit des Nickelsulfids im Entstehungszustande. ${ }^{1}{ }^{2}$ )

Bei der Methode Smith und Brunner ${ }^{3}$ ) wird die bei der Fällung des Zinksulfids freigewordene Mineralsäure durch Zufügen von Alkali- oder Ammonacetat gebunden und durch die weniger elektrolytisch dissozierte Essigsäure ersetzt, dadurch die Konzentration der Wasserstoffionen derart vermindert, so daß die Aasfällung des Zinksulfids eine vollständige wird.

Zimmermanis ${ }^{4}$ ) verwendet zur Trennung Ammonrhodanat, das nach TREaDWELL ${ }^{5}$ ) wahrscheinlich stark aussalzend auf die Fällung des $\mathrm{ZnS}$ wirkt und

TreadWell und G.H. Kramers ${ }^{6}$ ) führen in der "Aussalzmethode“ diese Trennung unter Anwendung von Ammonsalzen einer starken Mineralsäure durch.

Ich habe bei meinen Versuchen das neutrale Ammontartrat benützt und gefunden, daß sich dasselbe zur Trennung verwenden läßt, wenn man nicht zu viel Tartrat verwendet und bei $60-70^{\circ} \mathrm{C}$ den $\mathrm{H}_{2} \mathrm{~S}$-Strom rasch durchleitet.

1) Traedwold, Lehrbuch d. analyt. Chemie, I, I.

2) Gessner u. Thies, Z. anorg. Chem. 85-87, S. 1-57. Über Nickelund Kobaltsulfid. (Die scheinbare Anomalie im Verhalten des NiS gegen Säure.)

3) Chem. Zentralblatt 1895, 26.

4) Ann. d. Chem u. Pharm. 199 (1879), 3; 204 (1880), 226.

5) Traedweld, Lehrbuch d. analyt. Chemie 11, 7.

6) Traedweld, Lehrbuch d. analyt. Chemie II, 7 . 
Die Versuchsordnung ist folgende:

Die schwachsaure Lösung der beiden Metalle, welche bei einem Reaktionsvolumen von $150-200 \mathrm{ccm} 0,1 \mathrm{~g} \mathrm{Zn}$ und $0,1 \mathrm{~g} \mathrm{Ni}$ enthält, wird mit $\mathrm{Na}_{2} \mathrm{Co}_{3}$-Lösung neutralisiert, bis eine geringe Trübung entsteht. Diese Trübung bringt man durch Zusatz von verdünnter Salzsäure wieder zum Verschwinden. Hierauf werden auf je $100 \mathrm{ccm}$ Lösung $8-12 \mathrm{ccm} \mathrm{n} / 10-\mathrm{HCl}$ und im ganzen $0,5-1 \mathrm{~g}$ Ammontartrat zugesetzt und auf $60-70^{\circ} \mathrm{C}$ erwärmt. Nun leitet man in raschem Strom Schwefelwasserstoff bis zur Sättigung durch (etwa 6-10 Min.) und läBt auf dem Wasserbade von etwa $60^{\circ} \mathrm{C}$ absitzen. Nach vollständiger Klärung der Lösung wird heiß filtriert und der Niederschlag mit heißem, mit $\mathrm{H}_{2} \mathrm{~S}$-gesättigtem Wasser, das im Liter $1-2 \mathrm{~g}$ Tartrat enthält, gewaschen. Die ersten Anteile (etwa $30-50 \mathrm{ccm}$ ) fängt man gesondert auf und zieht sie nochmals durchs Filter. Der Zinksulfidniederschlag ist rein weiß, gut und rasch filtrierbar und enthält nur äußerst geringe Spuren von Nickel, so daß eine einmalige Fällung genügt. Der Niederschlag wird getrocknet und im RosE-Tiegel als Sulfid im Wasserstoffstrom bestimmt.

Beleganalysen: Verwendet wurde $\mathrm{ZnCl}_{2}$ und $\mathrm{ZnSO}_{4}$ puriss. Merk und $\mathrm{NiSO}_{4}$ puriss. Merk, kobaltfrei; in $50 \mathrm{~cm}$ der Lösung etwa $0,1 \mathrm{~g} \mathrm{Ni}$.

Der Nickelgehalt der verwendeten $\mathrm{NiSO}_{4}$-Lösung wurde nach 2 Methoden bestimmt:

1. Fällung des Ni als Nickeldimethylglyoxim nach TschuG AeFF- ${ }^{1}$ ) Brunck $^{2}$ ) aus schwcahammoniakalischer Lösung mit Dimethylglyoxim.

2. Bestimmung des Nickels als Metall durch Elektrolyse aus ammoniakalischer Lösung nach GrвBs $^{3}$ ) oder Rooт. ${ }^{4}$ )

Die Mittelwerte der beiden Bestimmungen dienen als ,berechneter" Vergleichswert. (Siehe Beleganalysen II.)

Ich habe nun weitere Versuche angestellt, um zu konstatieren, ob es notwendig ist mit heißem ammontartrathaltigem und mit $\mathrm{H}_{2} \mathrm{~S}$-gesättigtem Wasser oder bloß mit reinem, beißem, mit $\mathrm{H}_{2} \mathrm{~S}$ gesättigtem Wasser zu waschen und ob ein Aufwirbeln des Nieder-

1) L. TschugaefF, Berliner Ber. 38 (1905), 2520.

2) O. Bruner, Z. angew. Chem. 1907, S. 1844.

3) W. Gibbs, $Z$. analyt. Chem. 3 (1864), 334; vgl. auch Fresenius u BerG MANN, Z. analyt. Chem. 19 (1880), 320.

4) Root, Journ of Phys. Chem. 7 (1903), 428; 9 (1905), 1; Zentralblalt 1904, I, 50;1905, I, 833 . 
Beleganalysen. (1I.)

\begin{tabular}{|c|c|c|c|c|c|c|c|}
\hline$\underset{\text { berechnet }}{\mathrm{Zn}}$ & Gef. & $\begin{array}{c}\mathrm{Ni} \\
\text { berechnet }\end{array}$ & Gef. & $\begin{array}{l}\text { Zusätze } \\
\text { pon } \\
\text { Ammonil- } \\
\text { tarirat } \\
\text { in } g\end{array}$ & $\begin{array}{l}\text { Daner } \\
\text { der } \mathrm{H}_{2} \mathrm{~S}- \\
\mathrm{Zufubr} \\
\text { in Min. }\end{array}$ & $\begin{array}{c}\text { Fehler } \\
\text { des } \\
\mathrm{ZnS}-\mathrm{N} \\
\text { in } \mathrm{g} \mathrm{Zn}\end{array}$ & $\begin{array}{l}\text { Nickelgehalt } \\
\text { des } \\
\text { ZnS-Nieder- } \\
\text { schlages }\end{array}$ \\
\hline $\begin{array}{c}\left.0,1025 \mathrm{~g} \mathrm{Zn}^{1}\right) \\
,, \\
, " \\
, " \\
, " \\
, " \\
0,0513 \mathrm{~g} \mathrm{Zn} \\
0,1025 \mathrm{~g} \mathrm{Zn} \\
0,0110 \mathrm{~g} \mathrm{Zn} \\
, " \\
,, \\
,,\end{array}$ & $\begin{array}{l}0,1026 \\
0,1028 \\
0,1024 \\
0,1025 \\
0,1027 \\
0,1022 \\
0,1028 \\
0,1024 \\
0,0509 \\
0,0511 \\
0,1020 \\
0,0112 \\
0,0111 \\
0,0108 \\
0,0109\end{array}$ & $\begin{array}{c}0,0484 \mathrm{~g} \mathrm{Ni} \\
0,0973 \mathrm{~g} \mathrm{Ni} \\
, " \\
", \\
0,0484 \mathrm{~g} \mathrm{Ni} \\
0,020 \ddot{g} \mathrm{~g} \mathrm{Ni} \\
0,097 \ddot{3} \mathrm{~g} \mathrm{Ni} \\
, " \\
, " \\
,\end{array}$ & $\mid \begin{array}{l}0,04839 \\
0,0484 \\
0,0973 \\
0,0973 \\
0,0972 \\
0,0973 \\
0,0482 \\
0,0485 \\
0,0204 \\
0,0204 \\
0,0973 \\
0,0973 \\
0,0973 \\
0,0972 \\
0,0974\end{array}$ & $\begin{array}{l}1 \\
0,5 \\
0,75 \\
0,75 \\
0,5 \\
1 \\
0,5 \\
0,75 \\
0,5 \\
0,5 \\
1 \\
0,5 \\
1 \\
0,75 \\
0,5\end{array}$ & $\begin{array}{r}10 \\
8 \\
10 \\
10 \\
7 \\
10 \\
10 \\
10 \\
10 \\
10 \\
10 \\
10 \\
10 \\
10 \\
10\end{array}$ & $\begin{array}{c}+0,0001 \\
+0,0003 \\
-0,0001 \\
- \\
+0,0002 \\
-0,0003 \\
+0,0003 \\
-0,0001 \\
-0,0004 \\
-0,0002 \\
-0,0005 \\
+0,0002 \\
+0,0001 \\
-0,0002 \\
-0,0001\end{array}$ & $\begin{array}{c}\text { Spur } \\
\text { Ni-frei } \\
, " \\
0,000 \mathrm{I}^{\prime}-2 \mathrm{~g} \mathrm{Ni} \\
\text { Ni-frei } \\
0,00025 \mathrm{~g} \mathrm{Ni} \\
- \\
\text { Spuren v. Ni } \\
- \\
- \\
- \\
0,0001 \mathrm{~g} \mathrm{Ni}\end{array}$ \\
\hline
\end{tabular}

schlages am Filter unbedingt erforderlich ist. Ich fand, da $\beta$ es genügt, mit reinem, heißem, mit $\mathrm{H}_{2} \mathrm{~S}$-gesïttigtem Wasser nur aufzugießen, ohne den Niederschlag aufzuwirbeln, denn der ZnS-Niederschlag entbält bei dieser Arbeitsweise im Maximum 0,0002 $\mathrm{g} \mathrm{Ni}$, so daß für technische Analysen diese Arbeitsweise a usreichend gute Resultate liefert.

Ich verminderte weiter den Zusatz von Ammontartrat und $\mathrm{HCl}$ und konstatierte, daß bei einem Reaktionsvolumen von $150-200 \mathrm{ccm}$, Zusätze von $0,1-0,5 \mathrm{~g}$ Tartrat und $5-6 \mathrm{ccm} \mathrm{n} / 10-\mathrm{HCl}$ auf je $100 \mathrm{ccm}$ Lösung genügen, um eine vollständige Trennung der beiden Metalle zu erreichen. Die ZnS-Niederschläge werden bei abnehmendem Tartratzusatz immer feiner, lassen sich jedoch gut filtrieren und sind fast Ni-frei.

Beleganalysen. (III.)

\begin{tabular}{|c|c|c|c|c|c|c|c|}
\hline$\stackrel{\mathrm{Zn}}{\text { berechnet }}$ & Gef. & $\begin{array}{c}\mathrm{Ni} \\
\text { berechnet }\end{array}$ & Gef. & $\begin{array}{l}\text { Zusätze } \\
\text { von } \\
\text { Ammen- } \\
\text { tartrat } \\
\text { in } g\end{array}$ & $\begin{array}{l}\text { Dailer } \\
\text { der } I_{2} 8- \\
\text { Zufuhr } \\
\text { in XIin. }\end{array}$ & $\begin{array}{c}\text { Fehler } \\
\text { des } \\
\mathrm{ZnS} \\
\text { in } \mathrm{g} \mathrm{Zn}\end{array}$ & $\begin{array}{c}\text { Nickelgehalt } \\
\text { des } \\
\text { ZnS-Nieder- } \\
\text { schlages }\end{array}$ \\
\hline $0,1025 \mathrm{~g} \mathrm{Zn}$ & 0,1025 & $0,0973 \mathrm{~g} \mathrm{Ni}$ & 0,0973 & 0,4 & 10 & - & - \\
\hline $0,1018 \mathrm{~g} \mathrm{Zn}$ & 0,1015 & $0,1083 \mathrm{~g} \mathrm{Ni}$ & 0,1083 & 0,25 & 10 & $-0,0003$ & Spuren v. Ni \\
\hline , & 0,1017 & & 0,1083 & 0,4 & 10 & $-0,0001$ & \\
\hline & 0,1014 & & 0,1082 & 0,1 & 10 & $-0,0004$ & $0,0001 \mathrm{~g} \mathrm{Ni}$ \\
\hline $0,0508 \mathrm{~g} \mathrm{Zn}$ & 0,0513 & $0,0484 \mathrm{~g} \mathrm{Ni}$ & 0,0486 & 0,25 & 10 & $+0,0005$ & $\ldots$ \\
\hline $0,0206 \mathrm{~g} \mathrm{Zn}$ & 0,0207 & & 0,0484 & 0,25 & 10 & $+0,0001$ & $\begin{array}{l}\text { geringe Spur } \\
\text { von } \mathrm{Ni}\end{array}$ \\
\hline $0,0108 \mathrm{~g} Z \mathrm{Zn}$ & 0,0110 & $0,1083 \mathrm{~g} \mathrm{Ni}$ & 0,1082 & 0,1 & 10 & $+0,0002$ & $0,0001 \mathrm{~g} \mathrm{Ni}$ \\
\hline- & 0,0113 & & 0,1083 & 0,1 & 10 & $+0,0005$ & \\
\hline
\end{tabular}

1) Reaktionsvolumen bei $0,1 \mathrm{~g} Z \mathrm{Zn} 150-200 \mathrm{ecm}$, bei $0,05 \mathrm{~g} \mathrm{Zn} 100-150 \mathrm{ccm}$, bei $0,01 \mathrm{~g} \mathrm{Zn} 100 \mathrm{ccm}$. 
Wenn man bei der Trennung unter den sonst gleichen Bedingungen wie früher $2 \mathrm{~g}$ und mehr Ammontartrat verwendet und bei höherer Temperatur als $70^{\circ} \mathrm{C}$ Schwefelwasserstoff einleitet, so fällt ein Teil Nickels als Sulfid mit und auch dann, wenn man länger als 5 bis $7^{\prime}$ Schwefelwasserstoff bej $2 \mathrm{~g}$. Tartrat einleitet.

Grenzbedingungen für die Trennung bei $0,1 \mathrm{~g} \mathrm{Zn}$ und $0,1 \mathrm{~g} \mathrm{Ni}$ in der Lösung:

Nach Neutralisieren der Lösung mit $\mathrm{Na}_{2} \mathrm{CO}_{3}$ bis zur schwachsauren Reaktion, Zusatz von 12-15 $\mathrm{cem} \mathrm{n} / 10-\mathrm{HCl}$ und $2 \mathrm{~g}$ Ammontartrat, wird auf etwa $70^{\circ} \mathrm{C}$ erwärmt und $6-7^{\prime}$ Schwefelwasserstoff eingeleitet. Die Zinkfällung ist vollständig, das $\operatorname{ZnS}$ rein weiß und liefert noch einwandfreie Resultate.

Nach vorigem ist die Grenze für die einfache Trennung für obige Konzentration:

2 g Tartratzusatz,

$70^{\circ} \mathrm{C}$ Temperatur der Lösung,

7 Min. rasches Einleiten von Schwefelwasserstoff,

150 -200 ccm Reaktionsvolumen.

$$
\text { Beleganalysen für den Grenzfall (IV): }
$$

\begin{tabular}{|c|c|c|c|c|c|c|c|c|}
\hline $\mathrm{Zn}$ ber. & Gef. & $\mathrm{Ni}$ ber. & Gef. & $\begin{array}{c}\text { Ammon- } \\
\text { tartrat }\end{array}$ & Temp. & $\begin{array}{c}\text { Dauer } \\
\text { der } \mathrm{H}_{2} \mathrm{~S} \text { - } \\
\text { Zuleitung }\end{array}$ & Fehler & $\begin{array}{l}\text { Ni-Gehalt } \\
\text { des ZnS }\end{array}$ \\
\hline 1018 & 1015 & 1083 & 1081 & $2 g$ & $70^{\circ}$ & 7 Min. & -3 & $0,0002 \mathrm{~g} \mathrm{Ni}$ \\
\hline- & 1023 & - & 1080 & $2 \mathrm{~g}$ & $65-70^{\circ}$ & & +5 & 0,0003, \\
\hline 508 & 512 & - & 1082 & $2 \mathrm{~g}$ & $65-70^{0}$ & & +4 & 0,00025, \\
\hline - & 506 & _- & 1083 & $2 g$ & $70^{\circ}$ & 7 & -2 & - \\
\hline
\end{tabular}

Nickelbestimmung im Filtrat von ZnS:

Der Nickelgehalt des Filtrats wurde nach Wegkochen des Schwefelwasserstoffes entweder mit Dimethylglyoxim als Nickeldimethylglyoxim nach. TschUGaEFF-1)BRUNEK ${ }^{2}$ ) oder elektrolytisch als Metall nach Methode GiBBs ${ }^{3}$ ) oder Root $^{4}$ ) bestimmt.

Weitere Versuche:

Da die Hydrolyse des Ammontartrats ziemlich weitgehend ist ${ }^{5}$ ), nur etwa 5 mal geringer als bei Ammonacetat und bei dem Erwärmen

1) L. Tschugahef, Berliner Ber. 38 (1905), 2520.

2) O, BRUnek, $Z$. angew. Chem. 1907. 1844.

$\left.{ }^{3}\right)$ W. GIBBS, $Z$. analyt. Chem. 3 (1864), 334.

4) Rоот, Journ. of Phys. Chem. 7 (1903), 428; 9 (1905), 1; Zentralblatt 1904, I, 50; 1905, I, 833.

5) AbegG, Handbuch d. anorg. Chem. III, 3, S. 244; Ostwald u. RaIOH, Z. physikal. Chem. 2 (1888), 125. 
auf etwa $70^{\circ} \mathrm{C} \mathrm{NH}$ frei wird, der mit $\mathrm{H}_{2} \mathrm{~S}$ zu $\left(\mathrm{NH}_{4}\right)_{2} \mathrm{~S}$ gebunden, NiS ausfällen könnte, untersuchte ich reine neutrale Ni-Lösungen auf ihr Verhalten bei $70^{\circ} \mathrm{C}$ und verschiedenen Zusätzen von Ammontartrat. Es ergibt sich nun, daß bei einem Reaktionsvolumen von $150-200 \mathrm{ccm}$ folgende Zusätze von $\mathrm{HCl}$ notwendig sind, um die A usfällung von NiS auch bei längerem Einleiten von $\mathrm{H}_{2} \mathrm{~S}$ zu verhindern:

$\begin{array}{rlrlcc}0,1 \mathrm{~g} \text { Ni } & 0,1 \mathrm{~g} & \text { Tartrat } & 0,05-0,1 & \mathrm{ccm} & \mathrm{n} / 10-\mathrm{HCl} \\ & 0,5, " & , & 0,3-0,8 & , & , \\ 1,0, & , & 1,5-2,5 & , & , \\ & 2,0, & , & 5,0-7,0 & , & ,\end{array}$

Ich konnte auch feststellen, daß sich bei der Trennung des Zn von $\mathrm{Ni}$ aus einer Lösung, die etwa $2 \mathrm{~g}$ Ammontartrat enthält, beim Einleiten von $\mathrm{H}_{2} \mathrm{~S}$ sich NiS an den Berührungsstellen mit der Luft ausschied, und zwar besonders an der etwas stark erhitzten Becherglaswand knapp über dem Spiegel der Lösung und am unteren Ende des Schwefelwasserstoffzuleitungsrohres. Werden jedoch Becherwand und Einleitungsrohr vor dem Beginn der $\mathrm{H}_{2} \mathrm{~S}$-Zufuhr mit etwas kaltem destilliertem Wasser abgespält, so tritt auch an diesen Stellen keine NiS-Bildung auf.

Stellt man nun nach beendeter Fällung ( $2 \mathrm{~g}$ Ammontartrat, $70^{\circ} \mathrm{C}, 6-7^{\prime} \mathrm{H}_{2} \mathrm{~S}-$ Zufuhr) des Zinksulfids, das Becherglas auf ein Wasserbad, das eine höhere Temperatur als $60^{\circ} \mathrm{C}$, z. B. $80-90^{\circ} \mathrm{C}$ besitzt, so kann man bemerken, daß sich an dem stärker erwärmten Boden des Glases immer NiS ausscheidet, während der darüber gelagerte ZnS-Niederschlag rein weiß bleibt. Diese NiS-Bildung wird bei längerem Stehen immer stärker, ist direkt proportional der Zeit und Temperatur, so daß nach 12 stündiger. Dauer bis $70 \%$ des $\mathrm{Ni}$ als NiS ausgefällt ist. Hierbei bemerke ich ausdrücklich, daß die Lösung bei diesen Verhältnissen stets sauer reagierte.

Um den Einfluß von größerem Zusatz von $\mathrm{NH}_{4}$-Tartrat zu ermitteln, wurden noch folgende Versuche angestellt. Die Lösung, die etwa $0,1 \mathrm{~g} \mathrm{Zn}$ and $0,1 \mathrm{~g} \mathrm{Ni}$ als Sulfat und Chlorid in $150-200 \mathrm{ccm}$ enthält, wurde mit $15 \mathrm{ccm} \mathrm{n} / 10-\mathrm{HCl}$ und $3-10 \mathrm{~g}$ Ammontartrat versetzt, auf etwa $60-70^{\circ} \mathrm{C}$ erwärmt und 5 Min. lang $\mathrm{H}_{2} \mathrm{~S}$ eingeleitet. Der Niederschlag von ZnS war rein weiß (längeres Einleiten hätte Ausscheidung von NiS zur Folge) und die Lösung klar. Nun wurde in der Kälte stehen gelassen. Wieder trat NiS-Ausfällung. auf und zwar steigend mit dem Zusatz von $\mathrm{NH}_{4}$-Tartrat und mit der Zeit. Die Lösung wurde jedesmal geprüft und war stark sauer. 
Im ersten Falle dürfte durch die höhere Temperatur des Wasserbades und stärkere Erwärmung des Bodens des Becherglases in der knapp darüber befindlichen Niedersehlagsschicht durch Hydrolyse ${ }^{1}$ ) des Ammontartrats freigewordenes $\mathrm{NH}_{3} \mathrm{mit}_{2} \mathrm{~S}$ zu $\left(\mathrm{NH}_{4}\right)_{2} \mathrm{~S}$ zusammentreten und die Ausscheidung bedingen und die darüberlagernde Niederschlagsschicht die Einwirkung der Mineralsäure hemmen.

Im zweiten Falle ist die Möglichkeit vorhanden, daß sich durch die längere Berührung mit der Luft die leichtlösliche Form des NiS²) in die unlösliche Form umsetzt und ausfällt. Durch die Ausscheidung aus den Lösungspartien, die mit der Luft in Berührung sind und die durch das Fallen des frischgebildeten Niederschlags hervorgerufene Flüssigkeitsbewegung gelangen immer wieder neue Anteile an die Oberfläche, so daß sich durch diesen Vorgang die äußerst langsame Zunahme der NiS-Ausscheidung vielleicht erklären läßt. Bei diesen Versuchen lagert sich das ausgeschiedene NiS als schwarze Schicht über dem rein weißen Zinksulfidniederschlag.

\section{Die Trennung des Zinks von Nickel mit Diammonphosphat.}

Nachdem ich diese Arbeit abgeschlossen hatte, suchte ich noch andere Wege, um $\mathrm{Zn}$ von $\mathrm{Ni}$ zu trennen. Angeregt durch die $\mathrm{Be}$ merkung TraenwelLs ${ }^{3}$ ), daß die Bestimmung des Zinks als $\mathrm{ZnNH}_{4} \mathrm{PO}_{4}$ zu vorzüglichen Resultaten führt, studierte ich die Arbeiten von H. TAMM $^{4}$ ), welcher als erster diese Bestimmung empfiehlt und in der einschlägigen Literatur die Untersuchungen von LösEKaNN und Th. Merer ${ }^{5}$ ), M. Austin ${ }^{6}$ ) und besonders die Ausführung dieser Zinkfällung von H. D. DAKIN ${ }^{7}$.)

Ich habe für die frühere Arbeit für den Vergleichswert des Zinks nach dieser Methode von DakıN den Zinkgehalt der Lösung bestimmt und tadellos übereinstimmende Werte erhalten.

Traenweld ${ }^{8}$ ) beschreibt diese Methode wie folgt:

Man versetzt die saure Zinklösung in der Platin- oder Porzellanschale mit Ammoniak bis zur schwachsauren Reaktion (neutraler

1) Vgl. Abeqq, Handbuch d. anorg. Chemie III, 3, S. 244, Hydrolyse von Ammonsalzen.

2) Vgl. Gessner u. Thied, Z, anorg. Chem. 85-87, S. 1-57, Über Cound Ni-Sulfid.

3) Traedwewl, II. Bd.

4) H. TAMM, Chem. News 24, 148.

$\left.{ }^{5}\right)$ G. Lösezann u. Th. Meyer, Chem. Ztg. 1886, S. 729.

$\left.{ }^{6}\right)$ M. Austiv, Amer. Journ. Sci 1899; Z. anorg. Chem. 22 (1900), 212.

7) H. D. DAKIN, Z. analyt. Chem. 39 (1900), 273.

8) Traedweld, II. Bd. 
Lösung setzt man $2-3 \mathrm{~g} \mathrm{NH}_{4} \mathrm{Cl} \mathrm{zu}$ ), verdünnt mit $\mathrm{H}_{2} \mathrm{O}$ auf $150 \mathrm{~cm}$ und erbitzt auf dem Wasserbade. Zu der warmen Lösung setzt man $10 \mathrm{mal}$ so viel Ammonphosphat, als Zink vorhanden ist, zu. Das sich zuerst abscheidende Zinkammonphosphat ist amorph, verwandelt sich aber rasch in einen fein kristallinischen Niederschlag, und zwar findet diese Umwandlung um so rascher statt, je mehr Ammonsalz in der Lösung enthalten ist. Man erhitzt 1/4 Std. weiter, entfernt die Schale, filtriert nach kurzem Stehen durch Goock-Tiegel, wäscht mit 1\% iger heißer Phosphatlösung chlorfrei, dann mehrmals mit kaltem Wasser, trocknet bei $100-105^{\circ} \mathrm{C} 2 \mathrm{Std}$. im Trockenschrank und wägt (Faktor 0,3664). Oder man erhitzt nach Einstellen in einen größeren Pt-Tiegel zunächst offen, dann bedeckt mit voller Tekluflamme bis zur Gewichtskonstanz oder erbitzt das getrocknete $\mathrm{ZnNH}_{4} \mathrm{PO}_{4}$ langsam im elektrischen Ofen bis zur bellen Rotglut und wägt als Pyrophosphat (Falstor 0,4290).

P. ArtmanN ${ }^{1}$ ) prüft den nach dieser Methode erhaltenen Niederschlag von $\mathrm{ZnNH}_{4} \mathrm{PO}_{4}$ maßanalytisch und zwar durch Titration des darin enthaltenen Ammoniaks auf jodometrischem Wege. (Ich werde auf diese Arbeit weiter unten noch zurückkommen.)

G. LUFF ${ }^{2}$ ) klärt in seinen Ausführungen einzelne Punkte dieser Zinkbestimmung auf und schreibt unter Punkt I, Allgemeine Bemerkungen, wie folgt:

Die dem Magnesium verwandten Elemente $\mathrm{Zn}, \mathrm{Cd}, \mathrm{Hg}$, aber auch $\mathrm{Mn}, \mathrm{Ni}$, Co und vielleicht noch manch anderes Element (z. B. Beryllium) bilden, wie das Magnesium, mit $\left(\mathrm{NH}_{4}\right)_{2} \mathrm{HPO}_{4}$ unter bestimmten Bedingungen kristallinische Niederschläge von der Zusammensetzung $\mathrm{Me}^{\prime \prime} \mathrm{NH}_{4} \mathrm{PO}_{4} \cdot \mathrm{H}_{2} \mathrm{O}$, welche sich zur gewichtsanalytischen Bestimmung eignen. In neutraler Lösung fällt durch $\left(\mathrm{NH}_{4}\right)_{2} \mathrm{HPO}_{4}$ meist zunächst amorphes Triphosphat, das mit übersebüssigem $\left(\mathrm{NH}_{4}\right)_{2} \mathrm{HPO}_{4}$ in das kristalliniscbe Doppelsalz übergeht:

a) $3 \mathrm{Me}^{\prime \prime} \mathrm{Cl}_{2}+4\left(\mathrm{NH}_{4}\right)_{2} \mathrm{HPO}_{4} \rightarrow \mathrm{Me}_{3}{ }_{3}\left(\mathrm{PO}_{4}\right)_{2}+6 \mathrm{NH}_{4} \mathrm{Cl}+2 \mathrm{NH}_{4} \mathrm{H}_{2} \mathrm{PO}_{4}$,

b) $2 \mathrm{Me}_{3}{ }_{3}\left(\mathrm{PO}_{4}\right)_{2}+2\left(\mathrm{NH}_{4}\right)_{2} \mathrm{HPO}_{4} \rightarrow 3 \mathrm{Me}^{\prime \prime} \mathrm{NH}_{4} \mathrm{PO}_{4}+\mathrm{NH}_{4} \mathrm{H}_{2} \mathrm{PO}_{4}$,

c) $\mathrm{Me}^{\prime \prime} \mathrm{Cl}_{2}+2\left(\mathrm{NH}_{4}\right)_{2} \mathrm{HPO}_{4} \rightarrow \mathrm{Me}^{\prime \prime} \mathrm{NH}_{4} \mathrm{PO}_{4}+2 \mathrm{NH}_{4} \mathrm{Cl}+\mathrm{NH}_{4} \mathrm{H}_{2} \mathrm{PO}_{4}$.

Diese kristallinischen Niederschläge können nach Filtration und Auswaschung entweder bei etwa $100^{\circ} \mathrm{C}$ getrocknet und als

1) P. Artmann, Z. analyt. Chem. 1915, S. 87. Die Bestimmung des Zinks als $\mathrm{ZnNH}_{4} \mathrm{PO}_{4}$ und ihre Anwendung auf Trennungen.

2) G. LUFF, Zur Bestimmung des Zinks als Ammonzinkphosphat, Chem. Ztg. 77 (1921), 613. 
wasserfreies Salz gewogen werden (Gooor-Tiegel) oder man kann sie glühen und als Pyrophosphat zur Wägung bringen.

Weiter eingehend auf die Ausführungen obengenannter Arbeit bemerke ich, daß meine Erfahrungen mit den Angaben G. LuFrs in folgenden Punkten vollkommen übereinstimmen:

1. Das Arbeiten in der Porzellanschale oder im Becherglase führt zu gleich guten Werten.

2. Der $\mathrm{ZnNH}_{4} \mathrm{PO}_{4}$-Niederschlag enthält stets geringə Mengen $\mathrm{SiO}_{2}$ (entsprechend $0,1 \mathrm{~g} \mathrm{Zn}$ ).

3. Die Filtrate enthalten immer Spuren von Zink. (Das Zink im Filtrate wurde mit $\mathrm{H}_{2} \mathrm{~S}$ als $\mathrm{ZnS}$ gefällt und das $\mathrm{Zn}$ jodometrisch mit $\mathrm{n} / 100-$ Jodlösung bestimmt.)

4. Der $\mathrm{ZnNH}_{4} \mathrm{PO}_{4}$-Niederschlag kann im Gooch-Tiegel bis $180^{\circ} \mathrm{C}$ getrocknet werden, ohne daß Fehler durch Zersetzung auftreten.

In der Folge wurden alle Bestimmungen in Bechergläsern bei aufgelegtem Uhrglas durchgeführt und zwar entweder am Wasserbad oder über kleiner Flamme am Drahtnetze.

Dem folgenden etwas vorgreifend, möchte ich bemerken, daß die Umsetzung des amorphen Niederschlages rascher vor sich geht, wenn man zu der fast siedenden Lösung am Rührwerk die $\left(\mathrm{NH}_{4}\right)_{2} \mathrm{HPO}_{4^{-}}$ Lösung zusetzt, hierdurch die Lösung in ständiger Bewegung bleibt und mit kleiner Flamme erhitzt fast Siedetemperatur beibehält. Eventuelles Stoßen wird durch das Rühren verhindert.

Ich habe nun folgende Versuchsanordnung gewählt:

Zu der schwachsauren oder neutralen Zinklösung werden $1,5-3 \mathrm{~g} \mathrm{NH}_{4} \mathrm{Cl}$ zugesetzt, auf etwa $150 \mathrm{ccm}$ mit Wasser aufgefüllt, bis fast zum Sieden (Blasenwerfen) erhitzt und hierauf mit einer Lösung von $\left(\mathrm{NH}_{4}\right)_{2} \mathrm{HPO}_{4}$ gefällt und zwar mit 10-15facher Menge des Zinkgehaltes entsprechend. ${ }^{1}$ Der anfänglich amorphe Niederschlag setzt sich nach $1 / 4$ stündigem weiteren Erhitzen in die kristallinische Form um. (Bei Anwendung des elektrischen Rührers dauert die Umsetzung 5-10 Min.) Das $\mathrm{ZnNH}_{4} \mathrm{PO}_{4}$ wird nun mit $1 \%$ iger heiBer Phosphatlösung in den Gooor-Tiegel gespült, bis zum Verschwinden der Cl-Reaktion mit Phosphatlösung und hierauf mit kaltem Wasser gewaschen. Der Niederschlag wird bei $105-110^{\circ} \mathrm{C}$ getrocknet und als $\mathrm{ZnNH}_{4} \mathrm{PO}_{4}$ gewogen (Faktor $=0,3664$ ).

Die auf diesem Wege erhaltenen Resultate stimmen sehr gut untereinander und ergeben keine Differenz mit den Ergebnissen

1) Siehe weiter unten, variierte Phosphatzusätze.

$Z$ anorg. U. allg. Cbem. Bu. 122. 
der Zn-Fällung nach Methode DakıN. Hervorzubeben wäre, daß, wie früher erwähnt, die Umsetzung in der fast siedenden Lösung (besonders am Rührer) etwas rascber verläuft als am Wasserbad.

$\mathrm{Da}$ ich nun in den weiter unten angeführten Trennungen des Zinks von Nickel die Fällung des Zinks als $\mathrm{ZnNH}_{4} \mathrm{PO}_{4}$ anwende, suchte ich Reagenzien, die einesteils diese Fällung des Zinks nicht beeinträchtigen und andernteils das $\mathrm{Ni}$ als komplexes Salz in Lösung halten.

In der früher genannten Arbeit von DR. (G. LuFF ${ }^{1}$ ) wird aǹgeführt, daß auch Nickel aus schwachsauren oder neutralen Lösungen mit $\left(\mathrm{NH}_{4}\right)_{2} \mathrm{HPO}_{4}$ gefällt einen dem $\mathrm{ZnNH}_{4} \mathrm{PO}_{4}$ gleich zusammengesetzten Niederschlag von $\mathrm{NiNH}_{4} \mathrm{PO}_{4}$ liefert. Ich habe nun Versuche mit reinen Nickellösungen unter Zusatz verschiedener Neutralsalze durchgeführt und gefunden, daß die neutralen Ammonsalze der Weinsäure bzw. Rhodanwasserstoffsäure mit Nickel derart beständige Komplexsalze bilden, so daB bei Zusatz von $\left(\mathrm{NH}_{4}\right)_{2} \mathrm{HPO}_{4}$ auch in der Hitze keine oder nur geringe Umsetzung unter Bildung von $\mathrm{NiNH}_{4} \mathrm{PO}_{4}$ erfolgt.

Es ergibt sich, daß man etwa die 15-20fache Menge Ammontartrat bzw. Ammonrhodanid auf Nickel bezogen zu einer Nickellösung zusetzen $\mathrm{mu}$, bei der 'Trennung des $\mathrm{Zn}$ von $\mathrm{Ni}$ jedoch etwa $30-40$ fache Menge, um die Ausfällung des $\mathrm{Ni}$ als $\mathrm{NiNH}_{4} \mathrm{PO}_{4}$ zu verhindern.

Ebenso wie die erwähnten Ammonsalze eignen sich auch die neutralen Alkalitartrate $\left(\mathrm{KNaC}_{4} \mathrm{H}_{4} \mathrm{O}_{6}\right)$ und $\mathrm{K}_{2} \mathrm{C}_{4} \mathrm{H}_{4} \mathrm{O}_{6}$ zur Nickelkomplexsalzbildung. Die bei der Fällung des $\mathrm{ZnNH}_{4} \mathrm{PO}_{4}$ auftretenden Nachteile bei Verwendung von Alkalitartraten werde ich bei den diesbezüglichen Versuchen anführen.

\section{Vorversuche.}

In einer Reihe von Vorversuchen mit reinen Zn-Lösungen überprüfte ich den Einfluß der verschiedenen, gewählten Neutralsalze, den Zusatz von $\mathrm{NH}_{4} \mathrm{Cl}$ und des $\left(\mathrm{NH}_{4}\right)_{2} \mathrm{HPO}_{4}$ als Fällungsmittel. Durch diese Arbeiten ermittelte ich die Grenzwerte der einzelnen Reagenzienzusätze, urn bei der Trennung lösende Wirkungen und andere Fehlerquellen zu vermeiden.

$\mathrm{Da}$ die Ammonsalze der Phosphorsäure und Weinsäure der Affinität der Säuren entsprechend stärker hydrolytisch gespalten sind als das Ammonchlorid bzw. Ammonrhodanid, so ist auch die Wirkung auf den bei der Fällung gebildeten Niederschlag von Zink-

1) Dr. G. Lurr, Chem. Ztg. 77 (1921), 613. 
ammonphosphat eine verschiedene. Erstere wirken durch das bei der hydrolytischen Spaltung des $\left(\mathrm{NH}_{4}\right)_{2} \mathrm{HPO}_{4}$ und $\left(\mathrm{NH}_{4}\right)_{2} \mathrm{C}_{4} \mathrm{O}_{6} \mathrm{H}_{4}$ freiwerdende Ammoniak bei Verwendung relativ größerer Zusätze lösend auf den Niederschlag unter Bildung von Zinkammonkomplexverbindungen.

Ammonchlorid und Ammonrhodanid, welche in viel geringerem Maße der Hydrolyse unterworfen sind, wirken dementsprechend nicht lösend, geben jedoch bei hohen Zusätzen zu große Zinkwerte durch eingeschlossene Ammonsalze.

Die neutralen Tartrate (Seignettesalz und neutrales Kaliumtartrat) liefern ebenfalls zu hohe Ergebnisse durch okludierte Alkalisalze.

Verwendet wurden folgende Reagenzien:

Diammonphosphat pro analysi Merk,

Ammontartrat neutral Merk,

Seignettesalz pro analysi Merk,

Kaliumtartrat neutral puriss. Merk,

Ammonchlorid pro analysi Merk,

Ammonrhodanid pro analysi Merk.

Zuerst wurde der Einfluß von Diammonphosphat als Fällungsmittel bestimmt. In der Zinklösung wurde der Gehalt an Zink wie früher ermittelt.

Zusätze ron Diammonphosphat variiert (V):

\begin{tabular}{|c|c|c|c|c|c|}
\hline Zn berechnet & Gefunden & $\begin{array}{c}\text { Zusatz von } \\
\mathrm{NH}_{4} \mathrm{Cl}\end{array}$ & $\left(\mathrm{NH}_{4}\right)_{2} \mathrm{HPO}_{4}$ & Fehler & $\begin{array}{l}\text { Reaktions- } \\
\text { Volumen }\end{array}$ \\
\hline $\begin{array}{c}0,0939 \mathrm{~g} \\
, " \\
" \\
" \\
, \\
" \\
" \\
"\end{array}$ & $\begin{array}{c}0,0939 \\
9396 \\
939 \\
940 \\
937 \\
934 \\
931 \\
921 \\
918\end{array}$ & $\begin{array}{c}1,5 \mathrm{~g} \\
", \\
" \\
" \\
" \\
" \\
" \\
"\end{array}$ & $\begin{array}{r}1 \mathrm{~g} \\
1,5 \mathrm{~g} \\
2 \mathrm{~g} \\
3 \mathrm{~g} \\
4 \mathrm{~g} \\
5 \mathrm{~g} \\
6 \mathrm{~g} \\
8 \mathrm{~g} \\
10 \mathrm{~g}\end{array}$ & $\begin{array}{c}- \\
- \\
- \\
+1 \\
-3 \\
-5-6 \\
-8-9 \\
-18-19 \\
-21-23\end{array}$ & $\begin{array}{c}150 \mathrm{ccm} \\
", \\
", \\
", \\
200 " \mathrm{ccm} \\
", \\
",\end{array}$ \\
\hline
\end{tabular}

Alle Versuche wurden in Parallelproben durchgeführt. Bis zur 30 fachen Menge (bezogen auf Zink) von $\left(\mathrm{NH}_{4}\right)_{2} \mathrm{HPO}_{4}$ ergibt sich kein Fehler, bei Zusatz von mehr Phosphat tritt immer stärkerer Ammoniakgeruch auf und proportional steigende Iöslichkeit dẹs Niederschlages. 
Ammontartratzusätze variiert $\left(\mathrm{NH}_{4} \mathrm{Cl} 1,5 \mathrm{~g}\right.$, Phosphat $\left.1,5 \mathrm{~g}\right)(\mathrm{Vl})$ :

\begin{tabular}{|c|c|c|c|c|}
\hline Zn berechnet & Gefunden & Tartrat & Fehler & Reaktions-Vol. \\
\hline $\begin{array}{c}0,0935 \mathrm{~g} \\
, \\
" \\
" \\
: \\
, \\
", \\
, \\
" \\
" \\
,\end{array}$ & $\begin{array}{r}0,0935 \\
935 \\
935 \\
936 \\
934 \\
936 \\
936 \\
937 \\
935 \\
933 \\
924 \\
909\end{array}$ & $\begin{array}{l}1 \mathrm{~g} \\
2, " \\
3, " \\
4, " \\
5, " \\
6 ", \\
7, " \\
8, " \\
9, " \\
10 ", \\
15, " \\
20, "\end{array}$ & $\begin{array}{l}\ldots \\
\ldots- \\
\cdots \\
+1 \\
-1 \\
+1 \\
+1 \\
+2 \\
-2 \\
-2 \\
-11-14 \\
-24-28\end{array}$ & $\begin{array}{c}150 \mathrm{cmm} \\
", \\
", \\
", \\
200 " \mathrm{ccm} \\
", \\
", \\
250 " \mathrm{~cm} \\
, "\end{array}$ \\
\hline
\end{tabular}

Die Fällung des Zinks als $\mathrm{ZnNH}_{4} \mathrm{PO}_{4}$ aus ammontartrathaltiger Lösung liefert durchwegs gute Resultate, die Umsetzung des Niederschlages in die kristallinische liorm erfolgt etwas rascher als in der vorigen Versuchsreihe und ist derselbe leicht filtrierbar.

Kalium-Natrium-Tartratzusätze variiert (VII): $\left(1,5 \mathrm{~g} \mathrm{NH} \mathrm{NH}_{4} \mathrm{Cl}, 1,5 \mathrm{~g}\right.$ Phosphat).

\begin{tabular}{c|c|c|c|c}
\hline Zn berechnet & GefundenZ & KNa-Tartrat & Fehler & Reaktions-Vol. \\
\hline \hline $0,0935 \mathrm{~g}$ & 0,0935 & $1 \mathrm{~g}$ & - & $150 \mathrm{ccm}$ \\
$"$ & 0,0937 & $2,$, & +2 &, \\
$"$ & 0,0939 & 2, & +4 &, \\
$\Rightarrow$ & 0,0940 & 4, & +5 & $"$, \\
& 0,0944 & $5,$, & +9 &,
\end{tabular}

Kaliumtartrat in verschiedenen Mengen zugesetzt (VIII) '(1,5 g NH $\mathrm{NH}_{4} \mathrm{Cl}, 1,5 \mathrm{~g}$ Phosphat).

\begin{tabular}{c|c|c|c|c}
\hline Zn berechnet & Gefunden & Kaliumtartrat & Fehler & Reaktions-Vol. \\
\hline \hline 935 & 935 & $1 \mathrm{~g}$ & - & $150 \mathrm{ccm}$ \\
$"$ & 938 & $2 "$ & +3 & $"$ \\
, & 939 & $3 "$ & +4 & $"$ \\
$"$ & 941 & $4 "$ & +6 & $"$ \\
\hline & 945 & $5 \%$ & +10 & $"$
\end{tabular}

Die Resultate mit Alkalitartraten sind etwas zu hoch, da sich die Alkalisalze nicht leicht durch Waschen entfernen lassen und sich wahrscheinlich neben $\mathrm{ZnNH}_{4} \mathrm{PO}_{4}$ auch $\mathrm{ZnKPO}_{4}$ bzw. $\mathrm{ZnNaPO}_{4}$ bilden. Wenn man den feinkristallinischen Niedersehlag in verdünnter heißer Salzsäure löst, die Lösung mit Ammoniak bis zur schwachsauren Reaktion neutralisiert und dann nochmals fällt ${ }^{1}$ ) erhält man brauchbare, etwas hohe Werte.

1) Trawdwel, Lehrbuch d. analyt. Chemie II, S. 113. 
Zusätze von Ammonchlorid variert: (1,5 g Phosphat) (IX).

\begin{tabular}{|c|c|c|c|c|}
\hline Zn berechnet & Gefunden & $\mathrm{NH}_{1} \mathrm{Cl}$ & Febler & Reaktions-Vol. \\
\hline $\begin{array}{l}935 \\
" \\
" \\
" \\
",\end{array}$ & $\begin{array}{l}935 \\
935 \\
935 \\
936 \\
939\end{array}$ & $\begin{array}{c}1,5 \mathrm{~g} \\
3 \mathrm{~g} \\
5 \mathrm{~g} \\
7,5 \mathrm{~g} \\
10 \mathrm{~g}\end{array}$ & $\begin{array}{r}- \\
- \\
+\quad 1 \\
+24\end{array}$ & $\begin{array}{c}150 \mathrm{ccm} \\
", \\
" \prime \\
200 " \mathrm{com}\end{array}$ \\
\hline \multicolumn{5}{|c|}{$\begin{aligned} & \text { Zusätze von Ammonrhodanid geändert (X): } \\
& 1,5 \mathrm{~g} \mathrm{NH}_{4} \mathrm{Cl}, 1,5 \mathrm{~g} \text { Phosphat). }\end{aligned}$} \\
\hline
\end{tabular}

\begin{tabular}{c|c|c|c|c}
\hline Zn berechnet & Gefunden & Ammonrhodanid & Fehler & Peaktions-Vol. \\
\hline 935 & 934 & $2 \mathrm{~g}$ & -1 & $150 \mathrm{ccm}$ \\
$"$ & $\mathbf{9 3 5}$ & $\mathbf{4 g}$ & - & $"$ \\
$"$ & $\mathbf{9 3 5}$ & $\mathbf{6 g}$ & - & $"$ \\
$"$ & 936 & $7,5 \mathrm{~g}$ & $+\mathrm{I}$ & $\mathbf{2 0 0 "}$
\end{tabular}

Die Versuche mit $\mathrm{NH}_{4} \mathrm{Cl}$ und $\mathrm{NH}_{4} \mathrm{CNS}$ wurden ebenfalls teils am Wasserbad teils am Rührer ausgeführt. Resultate sind bei Anwendung von ztiviel Ammonsalz zu hoch durch eingeschlossenes Chlorid oder Rhodanid und zeigen die Niederschlage ebenso wie bei größeren Zusätzon von $\left(\mathrm{NH}_{4}\right)_{2} \mathrm{HPO}_{4}$ oder $\left(\mathrm{NH}_{4}\right)_{2} \mathrm{C}_{4} \mathrm{H}_{4} \mathrm{O}_{6}$ beim Filtrieren aus derart konzentrierten Salzlösungen die unangenehme Eigenschaft dés Durchgehens des Niederschlages bei Filtrieren und Waschen des Zinkammonphosphates.

Da nun durch diese Versuche festgestellt war, dab ein Zusatz von 5-7 g Ammontartrat oder Rhodanid keine Fehler verursacht, wandte ich bei den folgenden Trennungen höchstens diese Zusätze an. Die zugesetzte Menge Ammonphosphat war $15 \mathrm{mal}$ so groß als der Zinkgehalt der Lösung.

Da eine einmalige Trennung kein Ni-freies $/ \mathrm{nNH}_{4} \mathrm{PO}_{4}$ lieferte, wurde zweimal gefällt.

\section{Die quantitative Trennung des Zinks von Nickel mit Ammontartrat als Ni-Komplexsalzbildner. ${ }^{1}$ )}

Die Durchführung ist nun folgende: Zu der neutralen oder schwachsauren Lösung der beiden Metalle (verwendet wurde

1) P. Antmann, Z. analyt. Chem. 1915, S. 87. Ähnliche Versuchsbedingungen bei der Trennung von $\mathrm{CuZn}$ mit Ammontartrat. Ich bemerke, daß ich den Weg zu meiner Trennung ohne Kemntnis dieser Arbeiten fand. Ich hatte meine Ver- 
$\mathrm{ZnCl}_{2}$ Merk, $\mathrm{NiSO}_{4}$, (o frei Merk) setzt man 1,5-2 $\mathrm{g} \mathrm{NH} \mathrm{NH}_{4} \mathrm{Cl}$ und $3 \mathrm{~g}$ Ammontartrat zu, verdünnt mit Wasser auf $150-200 \mathrm{cem}$ und erhitzt zum Sieden, fällt mit 15-20facher für Zink berechneten Menge von Diammonphosphat. Der entstandene amorphe Niederschlag setzt sich am Wasserbad oder über kleiner Flamme in $1 / 4$ bis ${ }_{1 / 2}$ Std. in die kristallinische Form um. Der $\mathrm{ZnNH}_{4} \mathrm{PO}_{4}$-Niederschlag enthält jedoch noch geringe Mengen von $\mathrm{NiNH}_{4} \mathrm{PO}_{4}$, was sich durch stark blaugrüne Farbe kenntlich macht. Nach Filtrieren und Waschen mit $1 \%$ iger heißer $\left(\mathrm{NH}_{4}\right)_{2} \mathrm{HPO}_{4}$-Lösung, wird der Niederschlag am Filter in heißer verdünnter $\mathrm{HCl}$ (höchstens 2-3 cem konz. $\mathrm{HCl}$ ) gelöst und mit heißem Wasser in das Trennungsgefäß zurückgespült.

Die saure Lösung wird nun mit $\mathrm{NH}_{3}$ neutralisiert bis eine kleine Jrübung von ausgeschiedenem $\mathrm{ZnNH}_{4} \mathrm{PO}_{4}$ entsteht und diese Trübung durch Zusatz verdünnter $\mathrm{HCl}$ gelöst, so daß die Lösung schwach sauer reagiert. Je nach der Intensität der Blaufärbung des ersten Niederschlages werden nun $1-2 \mathrm{~g}$ Ammontartrat zugesetzt, zum Sieden erhitzt und die Fällung mit der 10-12fachen Menge von $\left(\mathrm{NH}_{4}\right)_{2} \mathrm{HPO}_{4}$ wiederholt.

Der nun ausfallende Niederschlag von $\mathrm{ZnNH}_{4} \mathrm{PO}_{4}$ ist rein weiß and Ni-frei. Enthält er nur ganz geringe Spuren von $\mathrm{Ni}$, so ist or in nassem Zustande blaßhimmelblau bis grünlichblau, so daß man aus der Färbung des $\mathrm{ZnNH}_{4} \mathrm{PO}_{4}$ einen Schluß auf Reinheit des Niederschlages bzw. Nickelgehalt desselben ziehen kann.

Tch habe jeden Niederschlag auf Anwesenheit von $\mathrm{Ni}$ geprüft und dabei gefunden, daß sich ganz geringe Spuren von $\mathrm{Ni}$ genau und scharf erkennen lassen, da die Intensität der Färbung des $\mathrm{ZnNH}_{4} \mathrm{PO}_{4}$ im nassen Zustande dem Nachweis des Nickels mit Dimethylglyoxim an Empfindlichkeit fast gleichkommt. Beim Trocknen verschwindet die Färbung oder geht in hellschmutziggelb über. Der Niederschlag wird bei $105-110^{\circ} \mathrm{C}$ getrocknet und als Zinkammonphosphat gewogen.

suche bereits Anfang September 1921 beendet und lernte erst im Februar 1922 obengenannte Arbeit kennen, als ich meine Trennungsmethode auch auf $\mathrm{CuZn}$ anwenden wollte. In seinen zusammenfassenden Schlußbemerkungen behält sich Verfasser die Anwendbarkeit dieser Methode auf Trennung des Zinks von $\mathrm{Ni}$ und $\mathrm{Hg}$ vor. $\mathrm{Da}$ ich in der einschlägigen Literatur bis jetzt keine weiteren Arbeiten Prof. Artmanns veröffentlicht fand, teilte mir Prof. Armuann auf meine diesbezügliche Anfrage mit, daß Dr. W. HARTMaNN bereits im Juni 1921 diese Trennung des $\mathrm{Zn}$ von Ni mit Ammontartrat ausarbeitete, ohne sie jedoch bis heute veröffentlicht zu haben. Hierdurch habe ich tatsächlich bis jetzt keinerlei Kenntnis über den Umfang und Arbeitsweise dieser Arbeiten erlangt. 
Trennung des Zinks von anderen Metallen.

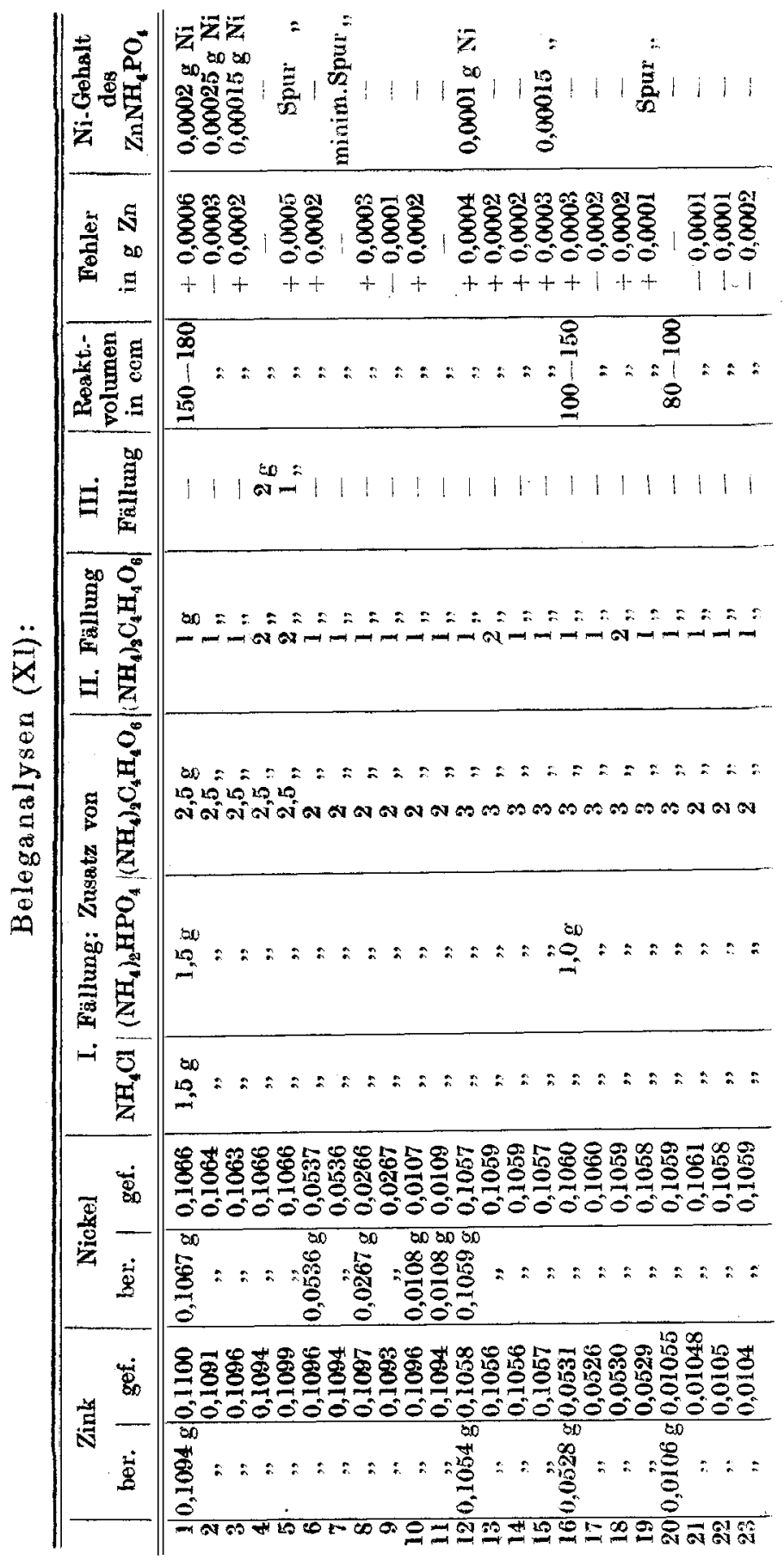


Ich habe weitere Versuche angestellt, ob durch oftmaliges Lösen und Fällen des $\mathrm{ZnNH}_{4} \mathrm{PO}_{4}$ bei der. Trennung Verluste entstehen und gefunden, daß selbst nach 5-10maliger Wiederholung nur ganz geringe Fehler auftreten.

Über die Bestimmung des Nickels im Filtrate werde ich weiter unten berichten.

Bei einigen Analysen wurde das erste und zweite Filtrat getrennt auf Ni-Gehalt untersucht. (XIa.)

\begin{tabular}{c|c|c|c}
\hline \hline Anal.-Nr. & I. Filtrat & II. Filtrat & Summe \\
\hline XIa 2 & $0,1022 \mathrm{~g} \mathrm{Ni}$ & $0,0042 \mathrm{~g} \mathrm{Ni}$ & $0,1064 \mathrm{~g} \mathrm{Ni}$ \\
6 & $0,0508, "$, & $0,0029, "$, & $0,0537, "$, \\
7 & $0,0521, "$, & $0,0015, "$, & $0,0536, "$, \\
11 & $0,0103, "$, & $0,0006, "$, & $0,0109, "$, \\
15 & $0,1031, "$, & $0,0026, "$, & $0,1057, "$, \\
17 & $0,1048, "$, & $0,0012, "$, & $0,1060, "$, \\
22 & $0,1054, "$, & $0,0004, "$, & $0,1058, "$,
\end{tabular}

Dem Folgenden vorgreifend, möchte ich bemerken, daß diese Trennung bei Anwendungen Ammontartrat die beste und rascheste der hier angeführten Trennungsmethoden ist. Die Umwandlung des amorphen Zn-Niederschlages in die kristallinische Form erfolgt in etwa $1 / 4-1 / 2$ Std., und ist derselbe leicht filtrierbar. Bei den Vorversuchen mit den Alkalitartraten und Ammonrhodanid war die Zeit der Umwandlung des $\mathrm{ZnNH}_{4} \mathrm{PO}_{4}$ in die kristallinische F'orm ziemlich gleich wie auch das Aussehen des Niederschlages. Bei den 3 folgenden Trennungen mit Alkalitartraten und Ammonrhodanid zeigt sich nun, daß bei Anwesenheit von $0,1 \mathrm{~g}$ Zn und $0,1 \mathrm{~g} \mathrm{Ni}$ in der Lösung die Umsetzung erst in etwa 2 Std. erfolgt (sowohl am Wasserbad wie auch am Rührer). Die Anwesenheit von viel $\mathrm{Ni}$ wirkt retardierend auf die Umwandlung, denn bei der zweiten Fällung, wo nur geringe Mengen $\mathrm{Ni}$ vorhanden sind, vollzieht sich dieser Prozeß viel rascher, so daß das Ende der Reaktion in $1 / 4-1 / 2$ Std. erreicht ist.

Da nach 1 stündigem Stehen am Wasserbad (oder Rührer) der stark amorphe Niederschlag sich gut abgesetzt hat, wenig $\mathrm{Ni}$ enthält und die überstehende Lösung ganz klar ist, filtrierte ich ab und wiederholte die Trennung, so daß ich hierdurch die Daner der Analyse verkürzte, ohne Fehler bei dieser Arbeitsweise zu erhalten, da das Zink vollständig gefällt war, nur geringe Mengen Ni enthält und sich heiß ziemlich rasch und gut filtrieren läßt. 


\section{Trennung des Zinks von Nickel mit Kalium-Natriumtartrat} (Seignettesalz) und neutralem Kaliumtartrat.

Die Versuchsanordnung ist die gleiche wie bei Verwendung von Ammontartrat als Komplexsalzbildner. Die Resultate ${ }^{1}$ ) von ZnNH ${ }_{4} \mathrm{PO}_{4}$ sind etwas zu hoch durch eingeschlossenes Alkali, das sich auch durch Anwendung von viel Waschwasser nicht gänzlich entfernen läBt. Um Verluste durch langes Waschen zu vermeiden, wird der Niederschlag, wie oben beschrieben, nochmals gefällt. Resultate nach dieser Methode zeigen nur geringe Fehler gegenüber der ersten mit Ammontartrat.

Beleganalysen: Zusätze von $1,5-3 \mathrm{~g} \mathrm{NH}_{4} \mathrm{Cl}, 1,5 \mathrm{~g}$ Phosphat bei $0,1 \mathrm{~g} \mathrm{Zn}, 1 \mathrm{~g}$ Phosphat bei $0,05 \mathrm{~g}$ Zn und geringerem Zinkgehalt. Die Reaktionsvolumina wie früher für die verschiedenen Konzentrationen.

\begin{tabular}{|c|c|c|c|c|c|c|}
\hline kerechnet & gefunden & $\begin{array}{r}\text { Nic } \\
\text { berechnet }\end{array}$ & $\begin{array}{l}\text { kel } \\
\text { gefunden }\end{array}$ & $\begin{array}{l}\text { Zusätze von } \\
\text { KNa-Tartrat }\end{array}$ & $\begin{array}{c}\begin{array}{c}\text { Fehler } \\
\text { in } \\
\mathrm{mg} / 10 \mathrm{Zn}\end{array} \\
\end{array}$ & $\begin{array}{c}\mathrm{Ni} \cdot \text { Gehalt des } \\
\mathrm{ZnNH}_{4} \mathrm{PO}_{4}\end{array}$ \\
\hline $\begin{array}{c}0,1091 \mathrm{~g} \\
, \\
0,05 \ddot{45} \mathrm{~g} \\
0,0108 \mathrm{~g} \\
0,1091 \mathrm{~g} \\
", \\
, "\end{array}$ & $\begin{array}{l}0,1088 \\
0,1092 \\
0,0549 \\
0,0540 \\
0,0106 \\
0,0109 \\
0,1093 \\
0,1095 \\
0,1092 \\
0,1089\end{array}$ & $\begin{array}{c}0,1012 \\
, \\
, \\
, \\
, \\
0,0507 \\
0,0100 \\
,\end{array}$ & $\begin{array}{c}0,1013 \\
0,1010 \\
0,1009 \\
0,1011 \\
0,1012 \\
0,1012 \\
506 \\
508 \\
0,0100 \\
0,0098\end{array}$ & $\begin{array}{ll}5 \mathrm{~g}, & 2 \\
3 \mathrm{~g} \\
3 \mathrm{~g}, & 2 \mathrm{~g} \\
3 \mathrm{~g}, & 1 \mathrm{~g} \\
3 \mathrm{~g}, & 1 \mathrm{~g} \\
3 \mathrm{~g}, & 1 \mathrm{~g} \\
3 \mathrm{~g}, & 1 \mathrm{~g} \\
2 \mathrm{~g}, & 1 \mathrm{~g} \\
3 \mathrm{~g}, & 1 \mathrm{~g} \\
3 \mathrm{~g}, & 1 \mathrm{~g} \\
2 \mathrm{~g}, & 1 \mathrm{~g} \\
2 \mathrm{~g}, & 1 \mathrm{~g}\end{array}$ & $\begin{array}{l}+4 \\
-3 \\
+1 \\
+4 \\
-5 \\
-2 \\
+1 \\
+2 \\
+4 \\
+1 \\
-2\end{array}$ & $\begin{array}{c}\text { Spur } \\
\overline{0} \\
0,00015 \mathrm{~g} \mathrm{Ni} \\
0,0002 \mathrm{~g} \mathrm{Ni} \\
0,000 \mathrm{I} \mathrm{g} \mathrm{Ni} \\
\frac{-}{-} \\
0,00005 \mathrm{~g} \mathrm{Ni} \\
- \\
-\end{array}$ \\
\hline
\end{tabular}

* Der $\mathrm{ZnNH}_{4} \mathrm{PO}_{4}$-Niederschlag lieferte nach der zweiten Füllung einen Zinkgehalt ron $0,1104 \mathrm{~g} \mathrm{Zn}$, nach Lösen und neuerlicher Fällung $0,1095 \mathrm{~g} \mathrm{Zn}$. Je höher die Alkalitartratzusätze waren, um so höher die Auswagen an $\mathrm{ZnNH}_{4} \mathrm{PO}_{4}$ und zwar bei $5 \mathrm{~g}$ and $2 \mathrm{~g}$ beträgt der Fehler in der Auswage $0,004-0,005 \mathrm{~g}$ als $\mathrm{ZnNH}_{4} \mathrm{PO}_{4}$, bei $4 \mathrm{~g}$ und $2 \mathrm{~g}$ bis $0,003-45 \mathrm{~g}$, bei $3 \mathrm{~g}$ und $2 \mathrm{~g}$ bis $0,0019-26 \mathrm{~g}$ und bei $2 \mathrm{~g}$ und $1 \mathrm{~g}$ bis $0,0016-21 \mathrm{~g}$.

\begin{tabular}{|c|c|c|c|c|c|c|}
\hline Zink & gefunden & \multicolumn{2}{|c|}{$\begin{array}{c}\text { Nickel } \\
\text { berechnet| gefunden }\end{array}$} & $\begin{array}{l}\text { Zusätze von } \\
\text { K-Tartrat }\end{array}$ & $\begin{array}{c}\text { Fehler } \\
\text { in } \\
\mathrm{mg} / 10 \mathrm{Za}\end{array}$ & $\begin{array}{l}\text { Ni-Gehalt } \\
\text { d. Ndschl. }\end{array}$ \\
\hline $0,1091 \mathrm{~g}$ & & & 0,1010 & $\mathrm{~g}, 2$ & +3 & 0,0002 \\
\hline 0.05 & & , & & $3 \mathrm{~g}$ & & \\
\hline & & ", & & $3 \mathrm{~g}$, & -1 & - \\
\hline 0,01 & & & & $\begin{array}{ll}3 \mathrm{~g}, & \mathrm{~g} \\
2 \mathrm{~g} & \mathrm{~g}\end{array}$ & $\begin{array}{l}+2 \\
+3\end{array}$ & 0,0001 \\
\hline & & 0,0100 & 0,0098 & $2 \mathrm{~g}, 1 \mathrm{~g}$ & & 0,0002 \\
\hline & 0,1093 & , & 0,0100 & $3 \mathrm{~g}, 1 \mathrm{~g}$ & +2 & - \\
\hline
\end{tabular}

1) Vgl. Bemerkung unter Fällung des Zinks aus reiner Zinklösung bei Anwesenheit von Alkalitartraten.

2) Die Mengen des zugesetziten Neutralsalzes bei der ersten und zweiten bzw. dritten Fällung stehen hintereinander: 


\section{Die Trennung des Zinks von Nickel mit Ammonrhodanid.}

Die folgenden Versuche mit Ammonrhodanid führten zu durchwegs guten Resultaten. Die Nachteile dieser Trennungsart sind wie früher erwähnt die längere Dauer der Umsetzung der ersten Fällung.

Die Versuchsanordnung ist gleich den obigen, die Behandlung des ersten Niederschlages wie bei Verwendung von .Alkalitartraten.

Beleganalysen: Zusätze von $\mathrm{NH}_{4} \mathrm{Cl}$ und $\left(\mathrm{NH}_{4}\right)_{2} \mathrm{HPO}_{4}$ und Reaktionsvolumen siehe Tabelle XI.

\begin{tabular}{|c|c|c|c|c|c|c|}
\hline berechnet & gefunden & $\begin{array}{r}\text { Nick } \\
\text { berechnet }\end{array}$ & gefunden & $\begin{array}{c}\text { Zusätze von } \\
\mathrm{NH}_{4} \mathrm{CNS}\end{array}$ & $\left|\begin{array}{c}\text { Fehler } \\
\text { in } \\
\mathrm{mg} / 10 \mathrm{Zn}\end{array}\right|$ & $\begin{array}{l}\mathrm{Ni} \text {-Gehalt des } \\
\mathrm{ZnNH}_{4} \mathrm{PO}_{4}\end{array}$ \\
\hline 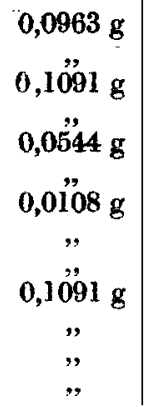 & $\begin{array}{l}0,0963 \\
0,0965 \\
0,1092 \\
0,1093 \\
0,0545 \\
0,0545 \\
0,01095 \\
0,0110 \\
0,0108 \\
0,1091 \\
0,1089 \\
0,1090 \\
0,1092\end{array}$ & $\begin{array}{c}1012 \\
\not 307 \\
1012 \\
507 \\
, " \\
1012 \\
, " \\
, " \\
0,0100 \\
0,0204\end{array}$ & $\begin{array}{r}1012 \\
1010 \\
507 \\
1009 \\
506 \\
507 \\
506 \\
1009 \\
1011 \\
1012 \\
1013 \\
100 \\
203\end{array}$ & $\begin{array}{l}4 \mathrm{~g}, 2,5 \mathrm{~g}, 2 \mathrm{~g} \\
4 \mathrm{~g}, 3 \mathrm{~g}, 2 \mathrm{~g} \\
4 \mathrm{~g}, 3 \mathrm{~g} \\
5 \mathrm{~g}, 3 \mathrm{~g}, 2 \mathrm{~g} \\
5 \mathrm{~g}, 3 \mathrm{~g} \\
4 \mathrm{~g}, 2 \mathrm{~g} \\
3 \mathrm{~g}, 3 \mathrm{~g} \\
4 \mathrm{~g}, 3 \mathrm{~g} \\
5 \mathrm{~g}, 2 \mathrm{~g}, 2 \mathrm{~g} \\
5 \mathrm{~g}, 3 \mathrm{~g}, 1 \mathrm{~g} \\
4 \mathrm{~g}, 3 \mathrm{~g}, 2 \mathrm{~g} \\
3 \mathrm{~g}, 1 \mathrm{~g} \\
3 \mathrm{~g}, 2 \mathrm{~g}\end{array}$ & $\begin{array}{l}- \\
+2 \\
+1 \\
+2 \\
+1 \\
+1 \\
+15 \\
+2 \\
- \\
-2 \\
-1 \\
+1\end{array}$ & $\begin{array}{c}0,0002 \text { g } \mathrm{Ni} \\
\overrightarrow{0} \\
0,00015 \\
\text { Spur } \\
\text { ", } \\
0,00025, " \\
- \\
- \\
\overrightarrow{-} \\
0,0001\end{array}$ \\
\hline
\end{tabular}

Ich habe auch hier bei einigen Analysen das erste und zweite Filtrat getrennt auf Nickelgehalt geprüft und ähnliche Verhältnisse wie früher erwähnt beobachtet, jedoch muB bei größerem Ni-Gehalt der Lösung dreimal gefällt werden, während bei geringen Ni-Mengen zweimalige Fällung ausreicht.

\section{Die Bestimmung des Nickels im Filtrat von $\mathrm{ZnNH}_{4} \mathrm{PO}_{4}$.}

Die Bestimmung des Nickels im Filtrat von $\mathrm{ZnNH}_{4} \mathrm{PO}_{4}$ habe ich nach folgenden Methoden durchgeführt:

1. Fällung des Nickels mit Dimethylglyoxim als Nickeldimethylglyoxim nach TschUG AEFF' ${ }^{1}$-BRUNok. ${ }^{2}$ )

Die Lösung wird in der Hitze mit 1\% iger alkoholiseher Dimethylglyoximlösung versetzt und Ammoniak bis zur alkalischen Reaktion zugefügt, der Niederschlag 2-3 std. absitzen lassen, hierauf durch Goosch-Tiegel filtriert, heiß gewaschen, bei $110-120^{\circ} \mathrm{C}$ getrocknet und gewogen.

2. Fällung des Nickels als sulfid mit farblosem Schwefelammon

1) I. Tschugakir, Berliner Ber. 38 (1905), 2520.

2) O. Bruvok, Z. angew. Chem. 1907, S. 1844. 
nach Ansäuern der Lösung mit Essigsäure.1) Das Nickelsulfid wird filtriert, in Königswasser gelöst, die Lösung mit Schwefelsäure abgeraucht und $\mathrm{Ni}$ elektrolytisch bestimmt.

3. Bestimmung des Nickels als Metall durch Elektrolyse. ${ }^{2}$ ) Die vereinigten Filtrate werden auf etwa $100-150 \mathrm{ccm}$ eingeengt, hierauf $30-50 \mathrm{ccm}$ konzentrierter Ammoniak zugesetzt und bei $60^{\circ} \mathrm{C}$ die Elektrolyse des Nickels durchgeführt. Die Dauer der Abscheidung beträgt bei 2,8-3,3 Volt Klemmenspannung und 0,8 bis $2 \mathrm{Amp}$. je nach dem Mengenverhältnis der in der Lösung befindlichen Ammonsalze 1-2 Std. Das ausgeschiedene Metall ist rein weiß und zeigt nur bei zu geringem Zusatz von Ammoniak Braunfärbung durch Bildung von Nickeloxydul.

\section{Zusammenfassung.}

1. Das neutrale Ammontartrat läßt sich sowohl zur Fällung des Zinks als Sulfid aus saurer Lösung wie auch zur Trennung des Zinks von Nickel mit Schwefelwasserstoff in mineralsaurer Lösung mit Erfolg verwenden und führt unter den früher angeführten Bedingungen bei einmaliger Durchführung der Fällung und Trennung zu brauchbaren Resultaten.

Wie schon erwähnt bildet das Ammontartrat mit Nickelsalzen ziemlich beständige komplexe Verbindungen. Ob nun bei dieser Treninung des $\mathrm{Zn}$ von $\mathrm{Ni}$ mit $\mathrm{H}_{2} \mathrm{~S}$ diese Eigenschaft des Tartrates gegenüber Nickel und die Beständigkeit der komplexen Nickelammontartrate gegen Schwefelwasserstoff oder blob der Ersatz der bei der Fällung freigewordenen Mineralsäure durch die weniger elektrolytisch dissozierte Weinsäure die Hauptrolle spielt, wäre noch $\mathrm{zu}$ untersuchen.

Zur Klarlegung dieser Frage, Anwendung und Übertragung der Methode auf Trennung von Co und $\mathrm{Mn}$, behalte ich mir diesbezügliche Arbeiten vor.

2. Zur Fällung des Zinks als $\mathrm{ZnNH}_{4} \mathrm{PO}_{4}$ aus schwachsaurer bzw. neutraler mit Neutralsalzen der Wein- und Rhodanwasserstoffsäure versetzten Lösung und hierauf basierende Trennungen von Nickel, führe ich folgendes an:

1) T'rendwald, Lehrbuch d. analyt. Chemie I, S. 156 und Gessnar u. Thicl, Z. anorg. Chem. 86 (1914), 1.

2) Vgl. Roor, Journ. of Phys. Chem. 7 (1903), 428; 9 (1905), 1; Zentrat. blatt 1904, I, 50; 1905, 1,833 . 
a) Die Fällung des Znals /n $\mathrm{NH}_{4} \mathrm{PO}_{4}$ mit 10 -30facher Menge $\left(\mathrm{NH}_{4}\right)_{2} \mathrm{HPO}_{4}$ für Zink bei Zusatz von $3-5 \mathrm{~g}$ Ammontartrat ergibt bei rascher Umsetzung des amorphen Niederschlages in die kristallinische Form gute, mit der Methode von DAkIN übereinstimmende Werte.

b) Ammonrhodanid und Ammonchlorid, ebenso auch die neutralen Alkalitartrate liefern durch Einschluß von Ammon- bzw. Alkalisalzen im Niederschlag (erstere bei 7-10 g Zusatz letztere schon bei $2-3 \mathrm{~g}$ ) etwas zu hohe Resultate, hindern aber in reiner Zinklösung in keiner Weise die Umsetzung des anfangs amorphen $\mathrm{ZnNH}{ }_{4} \mathrm{PO}_{4}$ - Niederschlages.

c) Nickelsalze werden durch Zusatz von Ammontartrat oder Ammonrhodanid zu ihren Lösungen in komplexe Ammonnickeltartrate bzw. Ammonnickelrhodanide, durch neutrale Alkalitartrate in die entsprechenden Nickelalkalitartrate übergeführt; diese komplexen Verbindungen sind ziemlich beständig und erfahren beim Zusatz von $\left(\mathrm{NH}_{4}\right)_{2} \mathrm{HPO}_{4}$ auch in der Hitze keine oder nur geringe Umsetzung unter Bildung von $\mathrm{NiNH}_{4} \mathrm{PO}_{4}$.

d) Bei der Trennung des Zn von Ni fällt ein Teil des Nickels mit dem $\mathrm{ZnNH}_{4} \mathrm{PO}_{4}$ mit, selbst bei Anwendung von 5-7 g Neutralsalz enthält der erste Zn-Niederschlag immer geringe Mengen Nickel, so daß doppelte Fällung notwendig ist. Durch die doppelte Fällung unter Verwendung geringerer Mengen von Ammonsalzen werden Fehler vermieden und zwar:

1. Teilweise Löslichkeit des Niederschlages durch das bei der Hydrolyse des Ammontartrates freigewordene Ammoniak unter Bildung von Zinkammonkomplexsalzen,

2. Okkludieren von Ammonrhodanid und hierdurch hervorgerufene hohe Analysenergebnisse und

3. Durchgehen des Niederschlages beim Filtrieren und Waschen des Zinkammonphosphates aus zu konzentrierten Salzlösungen.

e) Die Anwesenheit von größeren Nickelmengen behindert die rasche Umwandlung des Niederschlages bei Verwendung von Ammonrhodanid und Alkalitartraten und wirkt stark verzögernd, während geringer Nickelgehalt die Umsetzung nur in unerbeblichem Maße beeinflußt.

f) Der $\mathrm{ZnNH}_{4} \mathrm{PO}_{4}$-Niederschlag enthält nur Spuren von Nickel (größter Fehler $0,0003 \mathrm{~g} \mathrm{Ni}$ ) und liefert bei der Trennung gute Resultate. 
3. Wie durch eine Reihe von Vorversuchen bereits festgestellt, läßt sich diese Methode auch auf die Trennung des Ni von Co und Mn ïbertragen, deren genaue Durchführung ich mir vorbehalte.

Zum Schlusse ist es mir eine angenehme Pflicht, meinem verehrten Lehrer und Institutsvorstande, Herm Prof. Dr. Caru Frenzed, für die Anregung zu dieser Arbeit und das stete Wohlwollen, welches er mir bei der Durchführung derselben entgegenbrachte, meinen verbindlichsten Dank auszusprechen.

Brilnn, Laboratorium für anorganische, analytische und physikalische Chemie, deutsche technische Hochschule.

Bei der Redaktion eingegangen am 18. Mü\% 1929. 